\title{
Extensive High-Accuracy Thermochemistry and Group Additivity Values for Halocarbon Combustion Modeling
}

\author{
David S. Farina Jr., Sai Krishna Sirumalla, Emily J. Mazeau, and \\ Richard H. West* \\ Department of Chemical Engineering, Northeastern University, Boston, MA 02115, USA \\ E-mail: r.west@northeastern.edu
}

\begin{abstract}
Standard enthalpies, entropies, and heat capacities are calculated for 16,813 halocarbons using an automated high-fidelity thermochemistry workflow. This workflow generates conformers at density functional tight binding (DFTB) level, optimizes geometries, calculates harmonic frequencies, and performs 1D hindered rotor scans at DFT level, and computes electronic energies at G4 level. The computed enthalpies of formation for 400 molecules show good agreement with literature references, but the majority of the calculated species have no reference in the literature. Thus, this work presents the most accurate thermochemistry for many halocarbons to date. This new data set is used to train an extensive ensemble of group additivity values and hydrogen bond increment groups within the Reaction Mechanism Generator (RMG) framework. On average, the new group values estimate standard enthalpies for halogenated hydrocarbons within $3 \mathrm{kcal} / \mathrm{mol}$ of their G4 values. A significant contribution towards automated mechanism generation of halocarbon combustion, this research provides thermochemical data for thousands of novel halogenated species and presents a self-consistent set of halogen group additivity values.
\end{abstract}




\section{Introduction}

\section{Halogenated Hydrocarbons (HHCs)}

Halogenated hydrocarbons (HHCs) are commonly used as flame suppressants and refrigerant working fluids. The first generation of these compounds, chlorofluorocarbons (CFCs) and hydrochlorofluorocarbons (HCFCs), depleted the ozone layer and were banned worldwide under the Montreal Protocol in the 1980s. ${ }^{1}$ The second generation, hydrofluorocarbons (HFCs), are ozone-friendly but are currently being phased out due to their high global warming potentials (GWPs). ${ }^{2}$ Despite these controls on high-GWP HFC production, a recent study discovered that emissions of HFC-23 $\left(\mathrm{CHF}_{3}\right)$, a potent greenhouse gas, reached an historic high in $2018 \cdot .^{3]}$

To address these environmental concerns, several low-GWP HHC refrigerants and suppressants have been proposed. However, the chemical properties that make these HFCs more environmentally friendly also increase their flammability. ${ }^{[4}$ Therefore, the combustion properties of these proposed HHCs are of the utmost concern. Since experimental studies of these properties are complex and costly, predictive kinetic modeling of HHC combustion is crucial in screening proposed compounds in order to facilitate their innovation and implementation.

Understanding the complex chemistry of new compounds and predicting their combustion behavior under different conditions requires the compilation and simulation of detailed kinetic mechanisms (or microkinetic models) which often contain thousands of elementary reactions among hundreds of intermediate species. Building these models by hand is extremely challenging and error-prone due to the vast number of possible species and reactions to consider, sparse thermokinetic data available in the scientific literature, and biases of the human choosing which pathways to select. Thus, a tool that generates these models automatically by enumerating and evaluating the many potential pathways by which HHCs combust will be instrumental in screening the flammabilty of greener refrigerants and suppressants. 


\section{Reaction Mechanism Generator}

Reaction Mechanism Generator (RMG) is an open-source software package that automatically builds detailed kinetic models by proposing elementary reactions and estimating chemical properties (physical, thermochemical, kinetic, solvation, etc.) using a database of reaction templates, thermokinetic data, and estimation methods. $\frac{516}{5}$ These chemical properties are first sought in a database of known parameters, but are more commonly estimated using hierarchical decision trees. Thermochemical parameters $\left(\Delta_{f} H_{298 K}^{\circ}, S_{298 K}^{\circ}, C_{p}^{T}\right)$ are usually estimated using Benson's group additivity method ${ }^{7}$ for closed-shell species and the Hydrogen Bond Increment (HBI) scheme ${ }^{8}$ for radicals. RMG's group additivity values are derived from high fidelity experimental data supplemented with high level quantum chemistry data.

The success of RMG's rate-based algorithm in generating reliable kinetic models that capture all the essential chemistry in complex reacting systems depends heavily on the accuracy of thermokinetic parameters. As RMG was originally developed to study the kinetics of hydrocarbon combustion, its databases contain extensive, although not exhaustive, thermokinetic data for CHO chemistry. Since many short-lived intermediate species and elementary reactions are impossible to isolate and investigate experimentally, quantum chemistry methods are needed to calculate thermokinetic parameters. Recent progress on expanding RMG to model nitrogen, $\frac{9}{9}$ sulfur, $\frac{10}{20}$ and silicon $\frac{11}{11}$ has shown that quantum chemistry calculations are a viable approach to expand RMG's databases and estimation methods to new chemical systems. Thus, adding high-accuracy halogen thermochemical data to RMG's thermodynamic libraries and group additivity trees is essential to extending RMG to model halocarbon combustion.

\section{Thermochemistry for HHCs}

As kinetic modeling of HHCs has been been studied for many decades, thermochemical data

for these molecules and their derivatives have accumulated through various theoretical investigations (Table 1). With recent advances in ab-initio quantum chemistry methods and 
codes, these studies use high-level ab initio composite schemes and/or isodesmic reactions to calculate thermochemical properties such as $\Delta_{f} H_{298 K}^{\circ}$ within or close to chemical accuracy (1 kcal/mol). Included in many of these studies are group additivity values (GAVs), in particular the works of Bozzelli and coworkers. 12 Since conventional group additivity does not work well for chlorocarbons, Chen and Bozzelli introduced a modified scheme which uses nonnext-nearest neighbor interaction terms (NNNI) to account for the destabilizing interaction between electronegative chlorine atoms on adjacent carbons. $\frac{19}{19}$ This modified NNNI-GAV scheme was later extended to fluorine-fluorine interactions in HFCs ${ }^{12}$ and chlorine-oxygen interactions in chlorinated hydroperoxides. 13

Although there are highly accurate thermochemical data in the literature, the data are very sparse and only cover a very small fraction of chemical space containing $\mathrm{C}, \mathrm{H}, \mathrm{O}, \mathrm{F}$, $\mathrm{Cl}$, and $\mathrm{Br}$ chemistry. Since RMG explores a significant portion of this chemical space as it builds a mechanism, more HHC thermochemical data are needed to train RMG's group additivity schemes to improve its accuracy and reliability in estimating thermochemical properties. Thus, the focus of this work is not to more accurately calculate thermochemistry of previously studied molecules; instead, the objectives are: (1) to compute thermochemistry for a large and diverse set of halocarbons, and (2) derive an extensive ensemble of halogen GAVs with NNNIs within RMG's framework.

\section{Methods}

\section{The enum-halocarb4 dataset}

Due to a scarcity of thermochemical data for HHCs in the literature, a new dataset, enumhalocarb4, was compiled in this work. In order to obtain high coverage and diversity of $\mathrm{CHO}-(\mathrm{F}, \mathrm{Cl}, \mathrm{Br})$ chemical space, this dataset was created by "halogenating" a systematically

enumerated set of over $600 \mathrm{CHO}$ species containing up to 4 heavy atoms generated by Margraf et al. 31 This "halogenating" process involved systematic substitutions of halogen 
Table 1: Decades of quantum chemistry calculations for halocarbons. (GAVs- group additivity values, CC-high level coupled-cluster based model chemistry, NNNI-non-next-nearest neighbor interaction)

\begin{tabular}{|c|c|c|c|c|}
\hline Year & Species & Methods & GAVs & Ref \\
\hline 1996 & $\sim 100 \mathrm{C} 1$ and $\mathrm{C} 2 \mathrm{HFCs}$ & BAC-MP4 & & 20 \\
\hline 1998 & $\begin{array}{l}\text { fluorinated and chlorinated } \mathrm{C} 1 \text { and } \\
\mathrm{C} 2 \text { species }\end{array}$ & G2, G2(MP2), CBS-Q, CBS-4 & & 21 \\
\hline 1999 & 2-fluoro-2-methylpropane & G2(MP2) (isodemic reactions) & C/C3/F (with NNNI) & 12 \\
\hline 2000 & $\sim 120 \mathrm{C} 1$ and $\mathrm{C} 2 \mathrm{HFCs}$ & G3 & & 22 \\
\hline 2003 & chloroethyl hydroperoxides & $\begin{array}{l}\text { B3LYP /6-311+G(3df,-2p), } \\
\text { QCISD }(T) / 6-31 G(d, p), \\
\text { CBSQ//B3LYP/6-31G(d,p) }\end{array}$ & $\begin{array}{l}\mathrm{INT} / \mathrm{OO} / \mathrm{Cl}, \quad \mathrm{INT} / \mathrm{OO} / \mathrm{Cl} 2, \\
\text { INT/OO/CL3 NNNI terms }\end{array}$ & 13 \\
\hline 2009 & fluorine and chlorine oxides & $\mathrm{CC}$ & & 23 \\
\hline 2010 & halomethane derivatives & $\mathrm{CC}$ & & 24 \\
\hline 2014 & $\mathrm{C}_{2} \mathrm{H}_{\mathrm{x}} \mathrm{F}_{6-\mathrm{x}}(\mathrm{x}=0-5)$ & $\mathrm{CC}$ & & 25 \\
\hline 2015 & $14 \mathrm{C} 2-\mathrm{C} 4 \mathrm{HFCs}$ & $\begin{array}{l}\text { B3LYP, CBS-QB3, CBS-APNO, } \\
\text { M06, M06-2X, wB97X, G4, } \\
\text { G4(MP2)-6X, W1U (isodesmic } \\
\text { reactions) }\end{array}$ & $\begin{array}{lr}\mathrm{C} / \mathrm{C} / \mathrm{F} / \mathrm{H} 2, & \mathrm{C} / \mathrm{C} 2 / \mathrm{F} / \mathrm{H}, \\
\mathrm{C} / \mathrm{C} / \mathrm{F} 2 / \mathrm{H}, & \mathrm{C} / \mathrm{C} 2 / \mathrm{F} 2, \\
\text { (with NNNI) } & \mathrm{C} / \mathrm{C} / \mathrm{F} 3\end{array}$ & 14 \\
\hline 2016 & $\mathrm{FO}_{\mathrm{x}} \mathrm{H}$ and $\mathrm{FO}_{\mathrm{x}} \mathrm{F}(\mathrm{x}=1-3)$ & ccCA, G3, G3B3, M06, M06-2X & & 26 \\
\hline 2016 & $\begin{array}{l}\mathrm{CH}_{3-\mathrm{x}} \mathrm{F}_{\mathrm{x}} \mathrm{OH} \text { and } \\
(\mathrm{x}=1-3)\end{array}$ & $\begin{array}{l}\text { CBS-QB3, CBS-APNO, } \quad \text { G4 } \\
\text { (isodesmic reactions) }\end{array}$ & $\begin{array}{l}\mathrm{C} / \mathrm{F} / \mathrm{H} 2 / \mathrm{O}, \mathrm{C} / \mathrm{F} 2 / \mathrm{H} / \mathrm{O}, \mathrm{C} / \mathrm{F} 3 / \mathrm{O} \\
\text { and } \mathrm{HBI} \text { groups (with NNNI) }\end{array}$ & 15 \\
\hline 2017 & $\mathrm{C} 1-\mathrm{C} 2 \mathrm{HFC}$ derivatives and radicals & $\mathrm{CC}$ & & 27 \\
\hline 2018 & $\begin{array}{l}65 \mathrm{C} 1 \text { and } \mathrm{C} 2 \text { brominated halocar- } \\
\text { bons }\end{array}$ & $\mathrm{G} 3, \mathrm{G} 4, \mathrm{ccCA}, \mathrm{CCSD}(\mathrm{T}) / \mathrm{CBS}$ & & 28 \\
\hline 2018 & $\begin{array}{l}\text { fluorinated and chlorinated methane } \\
\text { and ethane derivatives }\end{array}$ & diet-HEAT, diet-HEAT-F12 & & 29 \\
\hline 2019 & organofluorine compounds & $\begin{array}{l}\text { CCSD(T)/aug-cc- } \\
\text { pvqz//B3LYP/def2-TZVP-D3(BJ) }\end{array}$ & & 30 \\
\hline 2019 & C2-C3 fluorinated aldehydes & $\begin{array}{l}\text { CBS-APNO, CBS-4M, CBS-QB3, } \\
\text { M06-2X, } \omega \text { B97X, B3LYP, G2, G3, } \\
\text { G4, W1U (isodesmic reactions) }\end{array}$ & $\begin{array}{l}\mathrm{CO} / \mathrm{C} / \mathrm{F}, \mathrm{C} / \mathrm{CO} / \mathrm{F} 3, \mathrm{C} / \mathrm{CO} / \mathrm{F} / \mathrm{H} 2, \\
\mathrm{C} / \mathrm{C} / \mathrm{CO} / \mathrm{F} / \mathrm{H}, \quad \mathrm{C} / \mathrm{C} / \mathrm{CO} / \mathrm{F} 2, \\
\mathrm{C} / \mathrm{C} / \mathrm{CO} / \mathrm{F} / \mathrm{H} \text { (with NNNI) }\end{array}$ & 16 \\
\hline 2019 & $\begin{array}{l}\text { 1,1,2-trifluoroethene and oxidized } \\
\text { derivatives }\end{array}$ & $\begin{array}{l}\text { B2PLYP, M06-2X, G3MP2B3, } \\
\text { CBS-QB3, CBS-APNO (isodesmic } \\
\text { reactions) }\end{array}$ & $\begin{array}{l}\mathrm{C} / \mathrm{C} / \mathrm{F} 2 / \mathrm{OH}, \mathrm{C} / \mathrm{C} / \mathrm{F} / \mathrm{H} / \mathrm{OH} \text { (with } \\
\mathrm{NNNI} \text { ) }\end{array}$ & 17 \\
\hline 2020 & $\begin{array}{l}\text { dimethyl and ethyl methyl fluori- } \\
\text { nated ethers }\end{array}$ & $\begin{array}{l}\text { MN15/cc-pVTZ, CBS-QB3, and } \\
\text { CBS-APNO }\end{array}$ & $\begin{array}{l}\mathrm{C} / \mathrm{C} / \mathrm{F} / \mathrm{H} 2, \\
\mathrm{C} / \mathrm{F} / \mathrm{H} 2 / \mathrm{O} \text { and } \mathrm{HBI} \text { groups } / \mathrm{H} / \mathrm{O},\end{array}$ & 18 \\
\hline
\end{tabular}


atoms $(\mathrm{F}, \mathrm{Cl}, \mathrm{Br})$ for hydrogens using RDKit. ${ }^{32}$ Molecules that contain more than one halogen element $\left(\mathrm{CF}_{3} \mathrm{Br}\right.$ for example) are referred to as "mixed-halogens" and molecules with only a single halogen element $\left(\mathrm{CF}_{4}\right.$ for example) are called "mono-halogens". To reduce the size of the dataset, enum-halocarb4 was pruned by removing:

- radical species with more than one unpaired electron

- mixed-halogens with more than 8 heavy atoms

- cyclic mixed-halogens

The chemical composition of the 16,813 molecules in the enum-halocarb4 is shown in Table 2 .

Table 2: Composition of 16,813 molecules in enum-halocarb4

\begin{tabular}{|l|r|r|r|r|r|}
\hline Composition & $\begin{array}{c}\text { Noncyclic } \\
\text { Closed }\end{array}$ & $\begin{array}{c}\text { Noncyclic } \\
\text { Radical }\end{array}$ & $\begin{array}{c}\text { Cyclic } \\
\text { Closed }\end{array}$ & $\begin{array}{c}\text { Cyclic } \\
\text { Radical }\end{array}$ & Total \\
\hline $\mathrm{CHOF}$ & 1,048 & 1,591 & 278 & 441 & 3,358 \\
$\mathrm{CHOCl}$ & 1,040 & 1,552 & 264 & 393 & 3,249 \\
$\mathrm{CHOBr}$ & 721 & 721 & 254 & 382 & 2,078 \\
$\mathrm{CHOFCl}$ & 852 & 923 & 0 & 0 & 1,775 \\
$\mathrm{CHOFBr}$ & 1,540 & 1,858 & 0 & 0 & 3,398 \\
$\mathrm{CHOClBr}$ & 932 & 1,108 & 0 & 0 & 2,040 \\
$\mathrm{CHOFClBr}$ & 447 & 468 & 0 & 0 & 915 \\
\hline Total & 6,580 & 8,221 & 796 & 1,216 & 16,813 \\
\hline
\end{tabular}

\section{Thermochemistry Workflow}

The automated thermochemistry workflow used to calculate high-level thermochemical parameters $\left(\Delta_{f} H_{298 K}^{\circ}, S_{298 K}^{\circ}, C p_{1000 K}^{\circ}\right)$ of the enum-halocarb4 dataset is shown in Figure 1 . First, a SMILES representation of the molecule is used to generate a molecular graph of the species using RMG. Then, the molecule is embedded with RDKit ${ }^{32}$ to create a $3 \mathrm{D}$ geometry. After embedding, conformers are investigated using the systematic conformer generation algorithm implemented in AutoTST. 33134 This algorithm explores conformers by rotating dihedrals in $120^{\circ}$ increments, varying cis/trans isomerism of double bonds, and alternating $\mathrm{R} / \mathrm{S}$ sterochemistry for chiral centers. Conformers are optimized in ASE ${ }^{35}$ using the dftb+ calculator ${ }^{36}$ with the halorg-0-1 parameter set. ${ }^{37}$ A unique set of conformers is gathered by discarding geometrically similar molecules with a root-mean-square deviation below $0.1 \AA$ of 


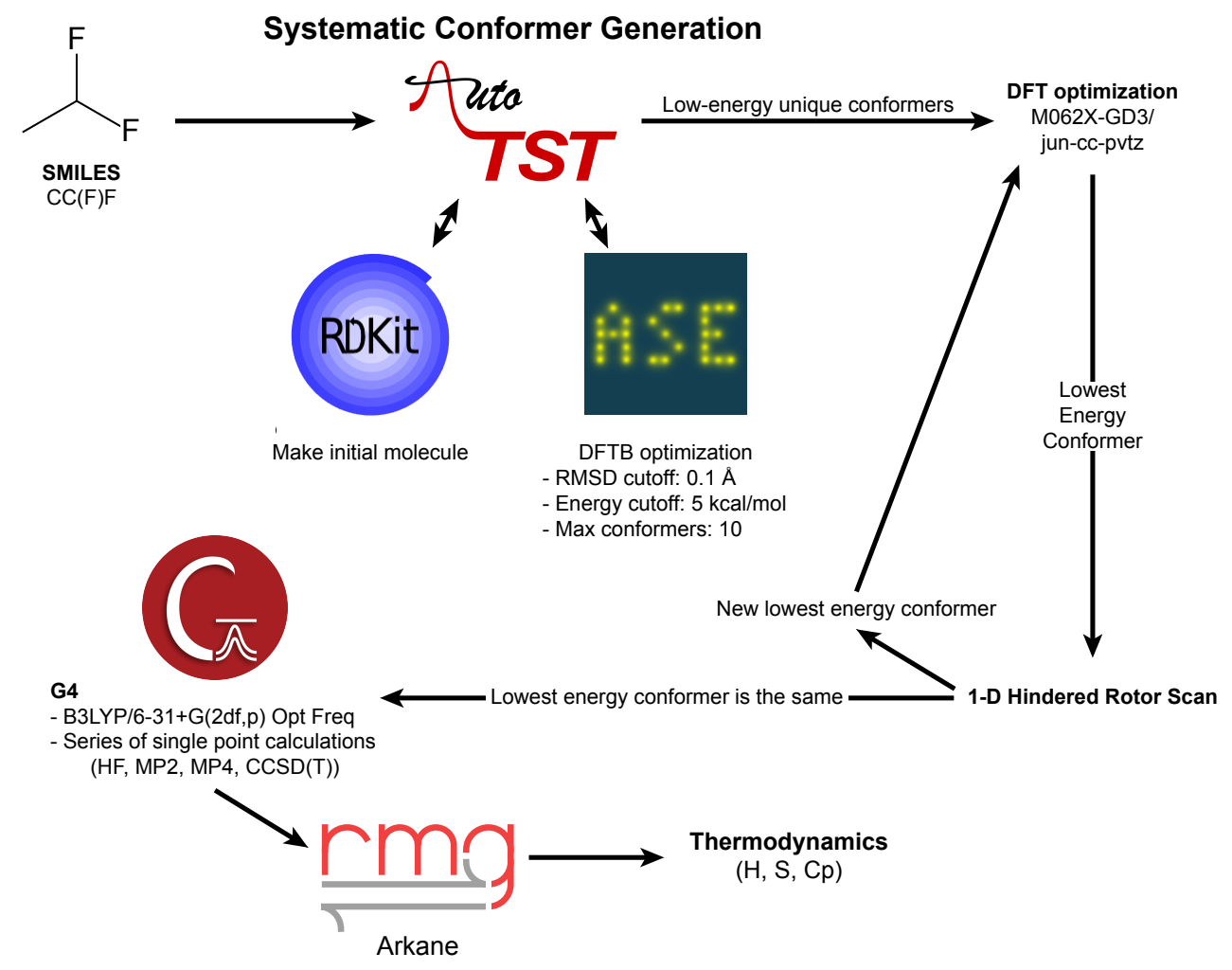

Figure 1: ab-initio Thermochemistry Workflow

a conformer in the unique set. Subsequently, to obtain a unique set of low-energy conformers, high energy conformers are discarded with electronic energies above $5 \mathrm{kcal} / \mathrm{mol}$ of the lowest energy conformer in the set. A maximum conformer cutoff of 10 was used in this work to limit the number of DFT calculations and save computational time.

These conformers are re-optimized with DFT in Gaussian 16 $6^{38}$ using M06-2X-D3/jun-ccpvtz (the M06-2X functional with Grimme's D3 empirical dispersion ${ }^{39}$ and the jun-cc-pvtz basis set $\left.{ }^{40}\right)$. Following all geometry optimizations, RMG's graph isomorphism algorithm is used to ensure that the optimized molecules match the corresponding input SMILES. If the optimized conformer is not isomorphic with the input molecular graph, the conformer is discarded from the set. If all of the optimized conformers were not isomorphic, that molecule was deemed unstable and removed from enum-halocarb4.

After identifying the lowest energy conformer with M06-2X-D3/jun-cc-pvtz, 1D hindered rotors calculations are performed in Gaussian 16 for molecules with internal rotors. For 
CHOF-containing molecules, the lowest energy conformer was optimized using B3LYP/6$31+\mathrm{G}(2 \mathrm{df}, \mathrm{p})$ and rotor scans were performed in $10^{\circ}$ increments with the same method. For molecules containing chlorine and bromine, the rotor scans were performed in $15^{\circ}$ increments using M06-2X-D3/jun-cc-pvtz. If a lower energy conformer is found during the rotor scans, the conformer is reoptimized starting with the lower energy coordinates with DFT and the rotor scans are redone. If the rotor scan is discontinuous, the rotor scan is rerun with all of the torsions frozen in the molecule except for the scan torsion. If the rotor scan is still discontinuous, the RRHO approximation is used.

To obtain more accurate electronic energies, the lowest energy conformer is re-calculated with the Gaussian 4 (G4) compound method ${ }^{41}$ in Gaussian 16. The G4 method was selected because it is an economical composite scheme that is accurate for halogenated hydrocar-

bons. $\stackrel{42 / 43}{ }$ Lastly, to obtain thermochemistry in NASA polynomial form, the G4 energies and harmonic frequencies and the DFT rotor scans are passed to RMG's statistical mechanics calculator Arkane, $\stackrel{44}{\underline{4}}$ a tool which uses quantum chemistry calculation results to compute thermochemical or kinetic parameters. For species with internal rotors, Arkane's best fit algorithm is used to determine whether a cosine function or a Fourier series better fits the energy profile of the rotor scan.

\section{Group Additivity Values}

The thermochemical data in the enum-halocarb4 dataset was used as training data to fit 2,041 new halogen thermo group addivitity values (GAVs) in RMG. Five types of thermo additivity groups were derived: conventional nearest-neighbor Benson groups, hydrogen bond increment groups (HBI), cyclic and noncyclic non-next-nearest neighbor interaction groups (long-distance), and ring correction groups. Table 4 shows the number of groups derived for each group type, and Table 3 demonstrates how these groups combine to estimate the enthalpy of formation for a hydrofluorocarbon radical $\mathrm{CH}_{3} \mathrm{CH}_{2} \mathrm{CHFCF}_{2}$ calculated in enumhalocarb4. 
Table 3: Group additivity enthalpy estimate for $\mathrm{CH}_{3} \mathrm{CH}_{2} \mathrm{CHFCF}_{2}$.

\begin{tabular}{|c|c|}
\hline Group & $\Delta_{f} H_{298 K}^{\circ}(\mathrm{kcal} / \mathrm{mol})$ \\
\hline \multicolumn{2}{|c|}{ Benson } \\
\hline Cs-CsHHH & -10.2 \\
\hline $\mathrm{Cs}-\mathrm{CsCsHH}$ & -4.9 \\
\hline $\mathrm{Cs}_{\mathrm{S}} \mathrm{Cs} \mathrm{CsFH}$ & -55.2 \\
\hline \multicolumn{2}{|c|}{ Long-Distance } \\
\hline $\mathrm{Cs}(\mathrm{F}) 2-\mathrm{Cs}(\mathrm{F})$ & +4.7 \\
\hline \multicolumn{2}{|c|}{ HBI } \\
\hline CsjF2(Cs-CsFH) & +100.2 \\
\hline $\mathrm{H}$ atom & -52.1 \\
\hline Total & -124.9 \\
\hline G4 & -126.2 \\
\hline Error & +1.3 \\
\hline
\end{tabular}

Table 4: Halogen GAVs derived in this work

\begin{tabular}{|l|r|}
\hline Type & Groups \\
\hline Benson & 226 \\
HBI & 1,421 \\
Ring Correction & 247 \\
Long-distance noncyclic & 91 \\
Long-distance cyclic & 56 \\
\hline Total & 2,041 \\
\hline
\end{tabular}

The new Benson groups were systematically created by generating every possible nearestneighbor group with either a carbon or oxygen center atom, bonded to at least one halogen atom. To maintain RMG's performance for estimating CHO thermochemistry, existing groups were held fixed while new halogen groups were fitted using scikit-learn's linear least square ridge regression method with an alpha of 1e-6. In total, 226 Benson groups and 91 long-distance interaction groups were derived using thermochemical data $\left(\Delta_{f} H_{298 K}^{\circ}, S_{298 K}^{\circ}\right.$, $C_{p}^{T}$ ) for 6,578 noncyclic closed-shell species in enum-halocarb4. To derive HBIs, closed-shell conjugates were created by saturating radicals with a hydrogen atom. Then, the HBIs were 
fitted by subtracting the thermochemical properties of the radical and hydrogen atom from the thermo of the saturated conjugate species as shown in equation 1, 3,825 radicals and their saturated closed-shell conjugates from enum-halocarb4 were used to fit 76 nearest-neighbor HBIs. To improve the accuracy of the HBI scheme, 1025 second nearest-neighbor HBIs and 320 three and four member ring HBIs were derived using 6,064 noncyclic radicals and 717 cyclic radicals, respectively.

$$
H B I(R \cdot)=R H-R \cdot-H \cdot
$$

Cyclic groups were also included to account for ring strain in halogen-substituted rings. 247 cyclic corrections and 56 long-distance cyclic interactions were derived from 796 closedshell three and four member ring species in enum-halocarb4. No corrections were included for ring sizes greater than four atoms since the maximum ring size in enum-halocarb4 is four.

\section{Test Set Generation}

To assess the accuracy of RMG's new halogen thermo groups in estimating thermochemistry of intermediates created during automated generation of HHC combustion models, an RMG model was constructed for 2-Bromo-3,3,3-trifluoropropene (2-BTP) and $\mathrm{CF}_{3} \mathrm{Br}$ in methane flames. Before generating a model, a literature mechanism for 2-BTP from NIST ${ }^{45}$ was imported into RMG. In order to teach RMG how these two flame suppressants behave in hydrocarbon flames, 727 of the 1,610 reactions in the literature mechanism were added as training reactions to $\mathrm{RMG}$ 's reaction families. Adding these reactions into training helps RMG generalize and improves rate estimates of reactions with similar functional groups. Then, an RMG model was built using the Foundational Fuel Chemistry Model Version 1.0 in RMG-database as a seed mechanism. enum-halocarb4 was used as an RMG thermo library during 2-BTP model generation, should RMG need thermochemistry for an intermediate in that dataset; other HHCs were estimated using the new GAV scheme. Our description is 
brief because the goal here is not to produce a better kinetic model, but to generate a test-set of molecules from a realistic scenario of automated model generation with RMG. 104 HHCs from the resulting RMG mechanism that were not in the enum-halocarb4 GAV training set were recalculated at G4 level using the automated thermochemisty workflow previously discussed, and the calculated thermochemical properties were compared to GAV estimates.

\section{Results and discussion}

\section{Thermochemistry Workflow Benchmark}

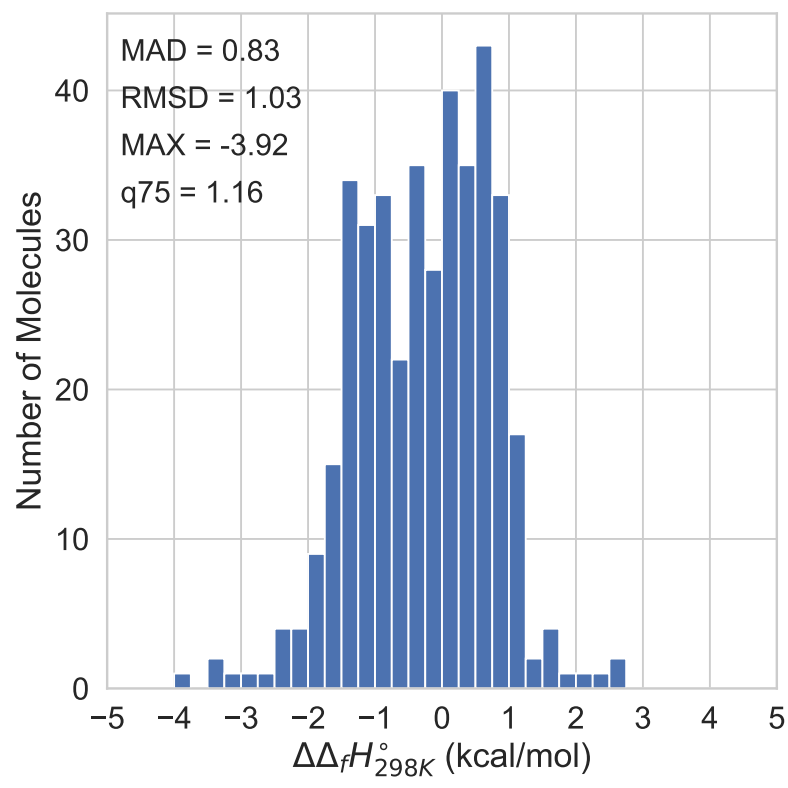

Figure 2: Enthalpies for halogenated species calculated at G4 level show good agreement with reference values. (MAD $=$ mean absolute deviation, $\mathrm{RMSD}=$ root-mean-square deviation, MAX $=$ maximum deviation, $\mathrm{q} 75=0.75$ quantile of absolute error)

To evaluate the accuracy of our automated workflow in calculating $\Delta_{f} H_{298 K}^{\circ}$ using the G4 method, 400 enum-halocarb4 species were benchmarked against literature data. Figure 2 shows the distribution of the error for the benchmark set compared to reference $\Delta_{f} H_{298 K}^{\circ}$

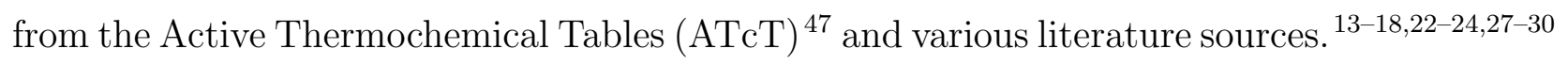
With a mean absolute error $(0.83 \mathrm{kcal} / \mathrm{mol})$ within chemical accuracy $(\leq 1 \mathrm{kcal} / \mathrm{mol}), \mathrm{G} 4$ 
is a suitable, relatively low cost composite quantum chemistry method for high-fidelity and high-throughput calculations of HHCs. However, for heavily halogenated systems, G4 and other composite methods do not compute enthalpies within chemical accuracy. ${ }^{48}$ Calculated G4 enthalpies for $\mathrm{C}_{2} \mathrm{Cl}_{5}$ and $\mathrm{C}_{2} \mathrm{Cl}_{6}$ in enum-halocarb4 are more than $3 \mathrm{kcal} / \mathrm{mol}$ lower than ATcT values. It is likely that heavily halogenated molecules in enum-halocarb 4 have higher errors than their more sparsely halogenated counterparts. To more accurately compute thermochemistry for heavily halogenated molecules, error-cancelling reactions, such as isodesmic reactions, could be used in place of the atomization approach used in this work. However, incorporating automated error-canceling reaction generation within our workflow was beyond the scope of the present work.

\section{Group Additivity Values Performance}

The new GAVs derived in this work dramatically improve RMG's estimates of $\Delta_{f} H_{298 K}^{\circ}$, $S_{298 K}^{\circ}, C p_{1000 K}^{\circ}$ for 16,456 species in enum-halocarb4, as shown in Figure 3. Overall, the mean absolute deviation (MAD) of RMG's GAV estimates for $\Delta_{f} H_{298 K}^{\circ}$ is reduced from $66 \mathrm{kcal} / \mathrm{mol}$ to $2.21 \mathrm{kcal} / \mathrm{mol}$ with the new groups, which is now on-par with RMG's CHO GAVs which typically estimate enthalpy within $2-3 \mathrm{kcal} / \mathrm{mol}$. The new groups also significantly improve accuracy at estimating $S_{298 K}^{\circ}(\mathrm{MAD}$ reduced from 92 to $1.48 \mathrm{cal} / \mathrm{mol} / \mathrm{K})$ and $C p_{1000 K}^{\circ}(\mathrm{MAD}$ reduced from 13 to $0.51 \mathrm{cal} / \mathrm{mol} / \mathrm{K})$. Based on the halogen GAV performance on the large and diverse enum-halocarb4 dataset, RMG's new halogen GAVs appear to be reliable at accurately predicting thermochemical properties for a wide range of halocarbons.

To further examine their accuracy and fidelity, the GAV performance with the new groups was evaluated for four different classes of molecules within the enum-halocarb4 dataset: noncyclic closed-shell, noncyclic radical, cyclic closed-shell, and cyclic radical. Figure 4 shows the GAV performance for each molecule class. For noncyclic HHCs, which are a majority of the dataset $(\sim 88 \%)$, the GAVs are accurate at estimating thermochemical parameters for both closed-shell molecules and radicals. For non-cyclic closed-shell molecules, the MAD for 

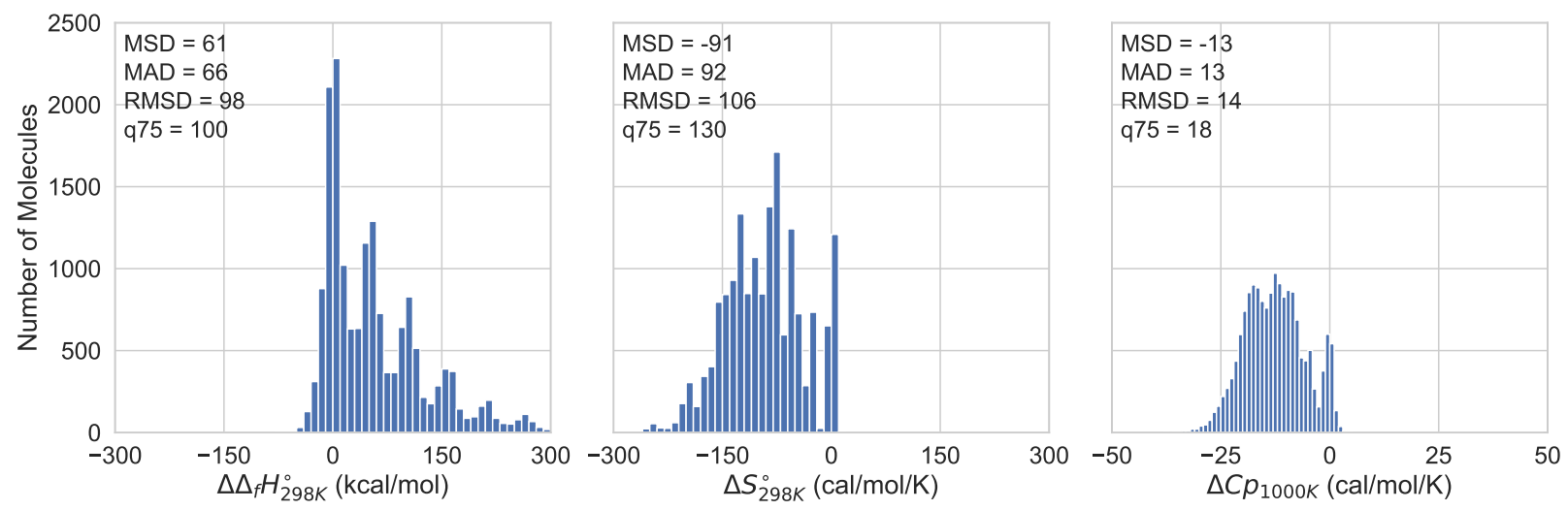

(a) RMG GAV estimates for enum-halocarb4 before new groups
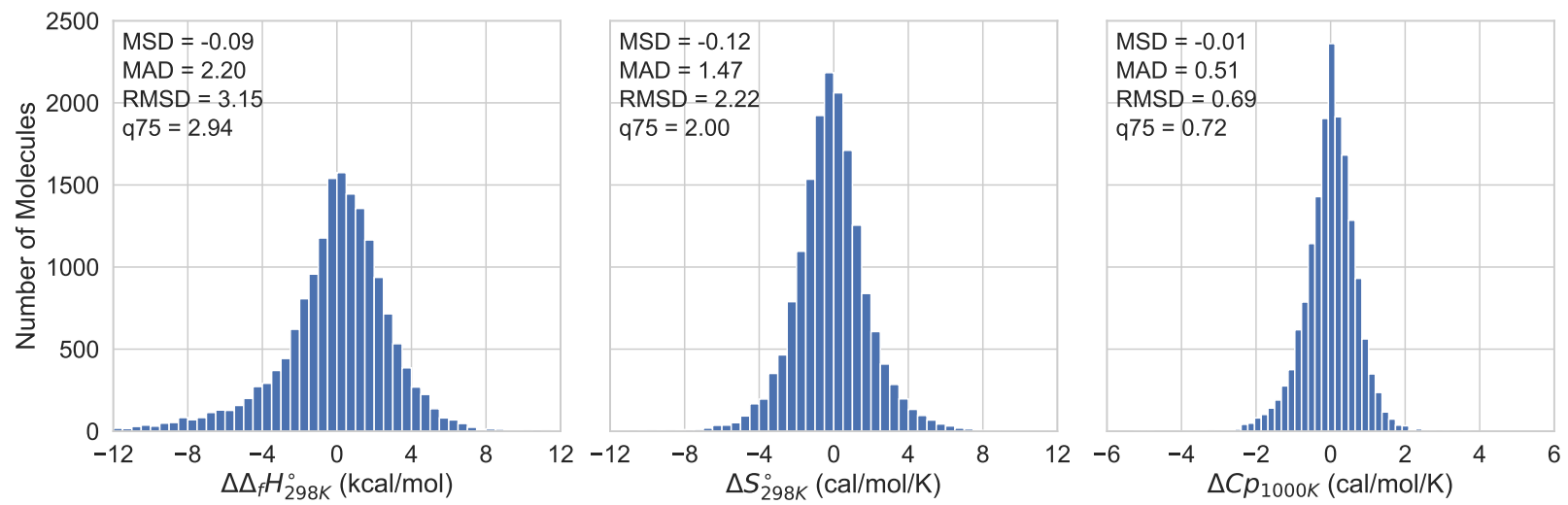

(b) RMG GAV estimates for enum-halocarb4 with new groups

Figure 3: New GAVs significantly improve RMG's estimates of $\Delta_{f} H_{298 K}^{\circ}, S_{298 K}^{\circ}, C p_{1000 K}^{\circ}$ for HHCs in enum-halocarb4computed using G4 level of theory. (MSD = mean signed deviation, $\mathrm{MAD}=$ mean absolute deviation, RMSD $=$ root-mean-square deviation, q75 = 0.75 quantile of absolute error) 


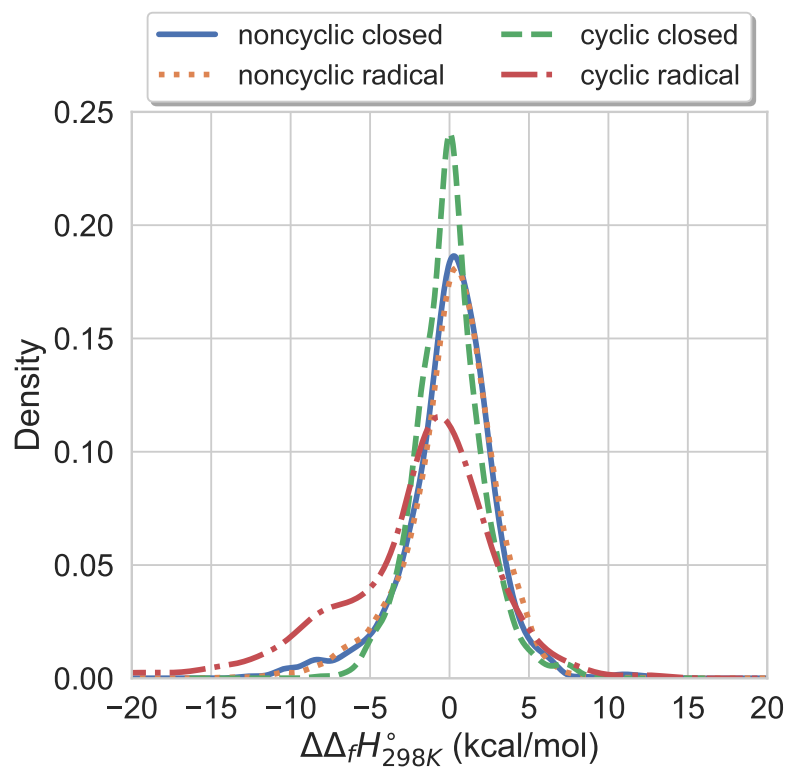

Figure 4: Probability density functions of GAV error for 4 classes of molecules in enumhalocarb4: noncyclic closed-shell, noncyclic radicals, cyclic closed-shell, cyclic radical molecules.

$\Delta_{f} H_{298 K}^{\circ}$ is $2.07 \mathrm{kcal} / \mathrm{mol}$ which is slightly less than the $2.13 \mathrm{kcal} / \mathrm{mol}$ MAD for noncyclic radicals. However, for cyclic species, the GAVs are significantly more accurate for cyclic closed-shell species $(\mathrm{MAD}=1.56 \mathrm{kcal} / \mathrm{mol})$ than for cyclic radicals $(\mathrm{MAD}=3.75 \mathrm{kcal} / \mathrm{mol})$. This disparity is most likely a result of only including closed-shell cyclic species in the derivation of the cyclic corrections and long-distance cyclic interaction groups. Therefore, since the radical cyclics are a test set for these groups whereas the closed-shell cyclics were included in training, it follows that the GAVs perform worse for the cyclic radicals.

Long-distance groups are included in the new GAV scheme to account for halogen/halogen and halogen/oxygen interactions on adjacent carbons. This modified group additivity approach was shown to significantly reduce errors in estimates of fluorinated and chlorinated hydrocarbons. ${ }^{12119}$ To investigate their impact in this work, the long-distance interactions were removed from the ensemble of GAVs, and estimated $\Delta_{f} H_{298 K}^{\circ}$ without these groups were compared to estimates with the groups included for molecules in enum-halocarb4 that have halogens on adjacent carbons. Figure 5 shows that, without the contributions from 


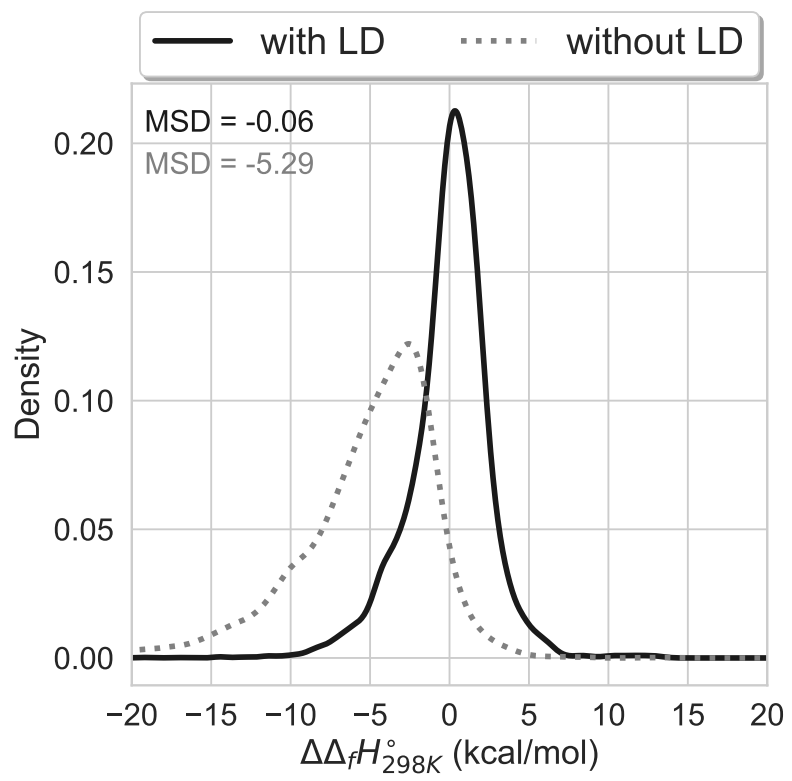

Figure 5: GAV enthalpy of formation estimates with long-distance groups (with LD in blue) and without long-distance interaction groups (without LD in red). Without long-distance groups, GAVs systematically underpredict enthalpies for HHCs.

long-distance interactions, the GAVs systematically underestimate the $\Delta_{f} H_{298 K}^{\circ}$ of these molecules by over $5 \mathrm{kcal} / \mathrm{mol}$ on average. In other words, GAVs overpredict stability of HHCs if lacking a long-distance term to capture the destabilizing interaction between halogens or oxygens on adjacent carbons. This indicates that these long-distance interactions are essential to accurately predict thermochemical properties of HHCs using a group additive scheme.

\section{RMG Test Set}

As automated mechanism generation is the intended application, the new halogen GAVs were implemented in RMG, and RMG was used to construct a test set. Since RMG explores a wide variety of molecules as it builds a mechanism, RMG molecules provide a challenging test for GAVs and other graph-based molecular property estimators. The performance of the GAVs for the 2-BTP RMG test set is shown in Figure 6. Expectedly, the GAVs showed poorer performance for estimating $\Delta_{f} H_{298 K}^{\circ}$ on the RMG test set with an overall 


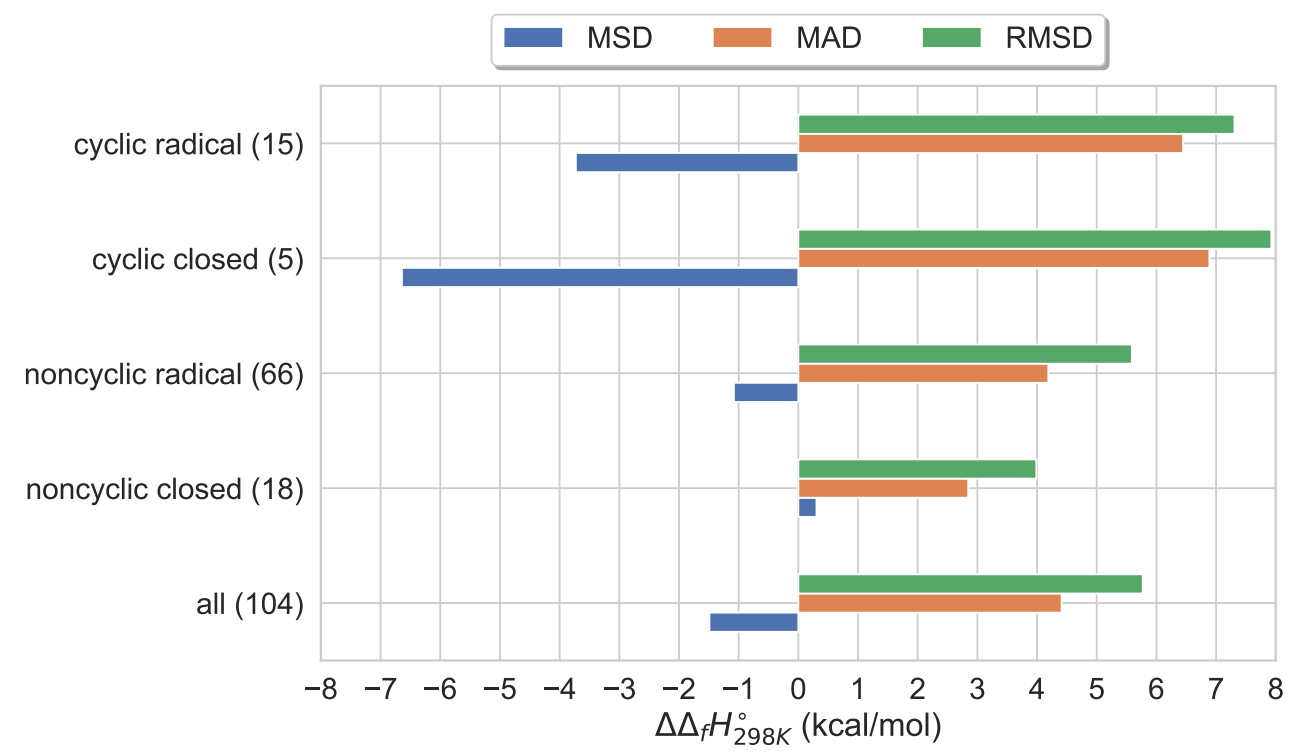

Figure 6: GAV $\Delta_{f} H_{298 K}^{\circ}$ estimates for $104 \mathrm{HHCs}$ in the 2-BTP RMG test set computed using G4 level of theory. The number of molecules in each category is indicated in parentheses. $(\mathrm{MSD}=$ mean signed deviation, $\mathrm{MAD}=$ mean absolute deviation, RMSD = root-meansquare deviation

mean absolute deviation of $4.69 \mathrm{kcal} / \mathrm{mol}$ compared to $2.21 \mathrm{kcal} / \mathrm{mol}$ on the enum-halocarb4 training set. The GAVs performed well for noncyclic test set molecules with mean absolute deviations of $2.85 \mathrm{kcal} / \mathrm{mol}$ for closed-shell species and $4.19 \mathrm{kcal} / \mathrm{mol}$ for radicals. However, the GAVs performed relatively poorly for cyclic compounds, with mean absolute deviations of $6.89 \mathrm{kcal} / \mathrm{mol}$ for closed-shell molecules and $6.44 \mathrm{kcal} / \mathrm{mol}$ for cyclic radicals. The poorer performance for the cyclic species can mainly be attributed to the following three factors. First, due to a lack of training data, the GAVs do not include halogen-specific ring corrections for ring sizes greater than 4 atoms. The mean absolute deviation for cyclic species with ring sizes greater than 4 was $7.42 \mathrm{kcal} / \mathrm{mol}$ compared to $6.09 \mathrm{kcal} / \mathrm{mol}$ for three and four member rings. Second, the long-distance corrections in RMG are not applied for neighboring atoms if one atom is in a ring and the other atom is outside the ring. A missing long-distance interaction between halogens on atoms in and out of a ring would lead to systematic underprediciton of $\Delta_{f} H_{298 K}^{\circ}$ for these types of molecules, which may be reflected in the negative mean signed deviation for cyclic compounds in the test set. Third, because 
RMG was used to build the test set and RMG prefers to incorporate low energy molecules in its models (endothermic reactions are typically slower), there is a bias for underestimated (i.e. lower energy) molecules to be selected for the test set. In other words, it is more likely for molecules that the GAVs underestimated (negative MSD) to end up in the test set than overestimated or correctly estimated molecules. Although the current GAV estimates do not perform as well for cyclic halocarbons as noncyclic ones, the estimates are good enough to be helpful in the automated construction of microkinetic models, and are a vast improvement on RMG's estimates before this work.

\section{Conclusions}

This research provides thermochemical data for thousands of novel halogenated species and presents a comprehensive, self-consistent set of halogen group additivity values within the Reaction Mechanism Generator framework. The new GAVs accurately estimate enthalpies of formation for noncyclic closed-shell and radical species in enum-halocarb4 and an RMG test set, but show poorer performance for rings for which more thermochemical training data are needed. Overall, the new halogen GAVs substantially improve RMG's thermo estimates for halocarbons, reducing the mean-absolute deviation of $\Delta_{f} H_{298 K}^{\circ}$ for the enumhalocarb4 dataset from 66 to $2.21 \mathrm{kcal} / \mathrm{mol}$. Importantly, these new groups will enable rapid and accurate on-the-fly estimation of halocarbon thermochemistry during automated model generation, thereby improving the fidelity and reliability of RMG's halocarbon combustion models for next-generation eco-friendly refrigerants and flame suppressants. This work also contributes a new data set, enum-halocarb4, which provides essential thermochemical data in a sparsely populated region of chemistry for training other machine learning estimation methods. 


\section{Acknowledgement}

This material is based upon work supported by the National Science Foundation under Grant No. 1751720. The authors acknowledge Information Technology Services, Research Computing at Northeastern University for providing high performance computing and storage, related software, visualization, and consulting resources. The authors thank Professor Bozzelli for sharing work on fluorine thermochemistry and GAVs, and also thank Dr. Linteris and Dr. Burgess for providing their $\mathrm{HFC}$ and $2-\mathrm{BTP} / \mathrm{CF}_{3} \mathrm{Br}$ kinetic models. Sai Krishna Sirumalla acknowledges funding from Entos Inc through LEADERs program at Northeastern University.

\section{Supporting Information Available}

The enum-halocarb4 dataset and group additivity values are publicly available in RMG's

open-source database (https://github.com/ReactionMechanismGenerator/RMG-database) and can also be accessed through the molecule search function on the RMG website (https: //rmg.mit.edu/molecule_search).

\section{References}

(1) Montreal Protocol on Substances that Deplete the Ozone Layer Final Act 1987. J. Environ. Law 1989, 1, 128-136, DOI: 10.1093/jel/1.1.128.

(2) Harby, K. Hydrocarbons and their mixtures as alternatives to environmental unfriendly halogenated refrigerants: An updated overview. Renew. Sust. Energ. Rev. 2017, 73, 1247-1264, DOI: $10.1016 /$ j.rser.2017.02.039.

(3) Stanley, K. M.; Say, D.; Mühle, J.; Harth, C. M.; Krummel, P. B.; Young, D.; O’Doherty, S. J.; Salameh, P. K.; Simmonds, P. G.; Weiss, R. F.; Prinn, R. G.; 
Fraser, P. J.; Rigby, M. Increase in global emissions of HFC-23 despite neartotal expected reductions. Nature Commun. 2020, 11, 397, DOI: 10.1038/ s41467-019-13899-4.

(4) Linteris, G.; Babushok, V. Numerically-Predicted Burning Velocities of C1 and C2 Hydrofluorocarbon Refrigerant Flames with Air. 17th International Refrigeration and Air Conditioning Conference at Purdue, West Lafayette, IN, 2018; https://tsapps. nist.gov/publication/get_pdf.cfm?pub_id=925585.

(5) Gao, C. W.; Allen, J. W.; Green, W. H.; West, R. H. Reaction Mechanism Generator: Automatic construction of chemical kinetic mechanisms. Comput. Phys. Commun. 2016, 203, 212-225, DOI: $10.1016 /$ j.cpc.2016.02.013.

(6) Liu, M.; Grinberg Dana, A.; Johnson, M. S.; Goldman, M. J.; Jocher, A.; Payne, A. M.; Grambow, C. A.; Han, K.; Yee, N. W.; Mazeau, E. J.; Blondal, K.; West, R. H.; Goldsmith, C. F.; Green, W. H. Reaction Mechanism Generator v3.0: Advances in Automatic Mechanism Generation. J. Chem. Inf. Model. 2021, 61, 2686-2696, DOI: 10.1021/acs.jcim.0c01480.

(7) Benson, S. W.; Cruickshank, F. R.; Golden, D. M.; Haugen, G. R.; O’Neal, H. E.; Rodgers, A. S.; Shaw, R.; Walsh, R. Additivity rules for the estimation of thermochemical properties. Chem. Rev. 1969, 69, 279-324, DOI: 10.1021/cr60259a002.

(8) Lay, T. H.; Bozzelli, J. W.; Dean, A. M.; Ritter, E. R. Hydrogen Atom Bond Increments for Calculation of Thermodynamic Properties of Hydrocarbon Radical Species. J. Phys. Chem. 1995, 99, 14514-14527, DOI: 10.1021/j100039a045.

(9) Dana, A. G.; Buesser, B.; Merchant, S. S.; Green, W. H. Automated Reaction Mechanism Generation Including Nitrogen as a Heteroatom. Int. J. Chem. Kinet. 2018, 50, 243-258, DOI: 10.1002/kin.21154. 
(10) Class, C. A.; Vasiliou, A. K.; Kida, Y.; Timko, M. T.; Green, W. H. Detailed kinetic model for hexyl sulfide pyrolysis and its desulfurization by supercritical water. Phys. Chem. Chem. Phys. 2019, 21, 10311-10324, DOI: 10.1039/C9CP00234K.

(11) Slakman, B. L.; Simka, H.; Reddy, H.; West, R. H. Extending Reaction Mechanism Generator to Silicon Hydride Chemistry. Ind $\&$ Eng Chem Res 2016, 55, 12507-12515, DOI: $10.1021 /$ acs.iecr.6b02402,

(12) Yamada, T.; Bozzelli, J. W. Thermodynamic Properties Hf $298^{\circ}, \mathrm{S}^{\circ} 298$, and $\mathrm{Cp}(\mathrm{T})$ for 2-Fluoro-2-MethyIpropane, Hf 298 of Fluorinated Ethanes, and Group Additivity for Fluoroalkanes. Phys. Chem. A 1999, 103, 7373-7379, DOI: 10.1021/jp9919516.

(13) Sun, H. Y.; Bozzelli, J. W. Structures, rotational barriers, and thermochemical properties of beta-chlorinated ethyl hydroperoxides. Phys. Chem. A 2003, 107, 1018-1024, DOI: $10.1021 / \mathrm{Jp} 022298 \mathrm{~g}$.

(14) Wang, H.; Álvaro Castillo,; Bozzelli, J. W. Thermochemical Properties Enthalpy, Entropy, and Heat Capacity of C1-C4 Fluorinated Hydrocarbons: Fluorocarbon Group Additivity. Phys. Chem. A 2015, 119, 8202-8215, DOI: 10.1021/acs.jpca.5b03912.

(15) Wang, H.; Bozzelli, J. W. Thermochemical Properties and Bond Dissociation Energies for Fluorinated Methanol, CH3-xFxOH, and Fluorinated Methyl Hydroperoxides, CH3xFxOOH: Group Additivity. Phys. Chem. A 2016, 120, 6998-7010, DOI: 10.1021/ acs.jpca.6b05293.

(16) Purnell, D. L.; Bozzelli, J. W. Thermochemical Properties: Enthalpy, Entropy, and Heat Capacity of C2-C3 Fluorinated Aldehydes. Radicals and Fluorocarbon Group Additivity. Phys. Chem. A 2019, 123, 650-665, DOI: 10.1021/acs.jpca.8b09065.

(17) Snitsiriwat, S.; Yommee, S.; Bozzelli, J. W. Thermochemistry of Intermediates and Products in the Oxidation Reaction of 1,1,2-Trifluoroethene via OH Radical. Phys. Chem. A 2019, 123, 8017-8027, DOI: 10.1021/acs.jpca.9b06647. 
(18) Hudzik, J. M.; Stoler, L. R.; Bozzelli, J. W.; Liebman, J. F. Thermochemistry of Fluorinated Dimethyl and Ethyl Methyl Ethers and Corresponding Radical Species. Chem. Eng. Data 2020, 65, 1594-1616, DOI: 10.1021/acs.jced.9b01034.

(19) Chen, C. J.; Wong, D.; Bozzelli, J. W. Standard chemical thermodynamic properties of multichloro alkanes and alkenes: A modified group additivity scheme. Phys. Chem. A 1998, 102, 4551-4558, DOI: 10.1021/jp980447i.

(20) Zachariah, M. R.; Westmoreland, P. R.; Burgess, D. R.; Tsang, W.; Melius, C. F. BACMP4 Predictions of Thermochemical Data for $\mathrm{C}_{1}$ and $\mathrm{C}_{2}$ Stable and Radical Hydrofluorocarbons and Oxidized Hydrofluorocarbons. J. Phys. Chem. 1996, 100, 8737-8747, DOI: $10.1021 / j p 952467 f$.

(21) Berry, R. J.; Schwartz, M.; Marshall, P. Computational Thermochemistry; Chapter 18, pp 341-358, DOI: $10.1021 / \mathrm{bk}-1998-0677 . \mathrm{ch} 018$.

(22) Haworth, N. L.; Smith, M. H.; Bacskay, G. B.; Mackie, J. C. Heats of formation of hydrofluorocarbons obtained by Gaussian-3 and related quantum chemical computations. Phys. Chem. A 2000, 104, 7600-7611, DOI: 10.1021/jp000475c.

(23) Karton, A.; Parthiban, S.; Martin, J. M. Post-CCSD(T) ab initio Thermochemistry of Halogen Oxides and Related Hydrides XOX, XOOX, HOX, XO, and HXO, (X = F, Xl), and Evaluation of DFT Methods for These Systems. Phys. Chem. A 2009, 113, 4802-4816, DOI: $10.1021 / \mathrm{jp} 8087435$.

(24) Csontos, J. J.; Rolik, Z. Z.; Das, S.; Kállay, M.; Kállay, M. High-Accuracy Thermochemistry of Atmospherically Important Fluorinated and Chlorinated Methane Derivatives. Phys. Chem. A 2010, 114, 13093-13103, DOI: 10.1021/jp105268m.

(25) Nagy, B.; Csontos, B.; Csontos, J.; Szakács, P.; Kállay, M. High-accuracy theoretical thermochemistry of fluoroethanes. Phys. Chem. A 2014, 118, 4824-4836, DOI: 10. 1021/jp503492a. 
(26) Alsunaidi, Z. H.; Wilson, A. K. DFT and ab initio composite methods: Investigation of oxygen fluoride species. Comput. Theor. Chem. 2016, 1095, 71-82, DOI: 10.1016/ j.comptc.2016.08.024.

(27) Ádám Ganyecz,; Kállay, M.; Csontos, J. Accurate Theoretical Thermochemistry for Fluoroethyl Radicals. Phys. Chem. A 2017, 121, 1153-1162, DOI: 10.1021/acs.jpca. $6 \mathrm{~b} 12404$

(28) Jorgensen, K. R.; Cadena, M. Theoretical study of bromine halocarbons: Accurate enthalpies of formation. Comput. Theor. Chem. 2018, 1141, 66-73, DOI: 10.1016/j. comptc.2018.08.016.

(29) Ganyecz, Á.; Kállay, M.; Csontos, J. High Accuracy Quantum Chemical and Thermochemical Network Data for the Heats of Formation of Fluorinated and Chlorinated Methanes and Ethanes. Phys. Chem. A 2018, 122, 5993-6006, DOI: 10.1021/acs. jpca.8b00614.

(30) Paulechka, E.; Kazakov, A. Critical Evaluation of the Enthalpies of Formation for Fluorinated Compounds Using Experimental Data and High-Level Ab Initio Calculations. Chem. Eng. Data 2019, 64, 4863-4874, DOI: 10.1021/acs.jced.9b00386.

(31) Margraf, J. T.; Reuter, K. Systematic Enumeration of Elementary Reaction Steps in Surface Catalysis. ACS Omega 2019, 4, 3370-3379, DOI: 10.1021/acsomega.8b03200.

(32) Landrum, G. RDKit: Open-source cheminformatics. http://www.rdkit.org.

(33) Bhoorasingh, P. L.; Slakman, B. L.; Seyedzadeh Khanshan, F.; Cain, J. Y.; West, R. H. Automated Transition State Theory Calculations for High-Throughput Kinetics. J. Phys. Chem. A 2017, 121, 6896-6904, DOI: 10.1021/acs.jpca.7b07361, PMID: 28820268 . 
(34) Harms, N.; Underkoffler, C.; West, R. Advances in automated transition state theory calculations: improvements on the AutoTST framework. ChemRxiv Preprint 2020, DOI: $10.26434 /$ chemrxiv.13277870.

(35) Larsen, A. H., et al. The atomic simulation environment - a Python library for working with atoms. J. Condens. Matter Phys. 2017, 29, 273002, DOI: 10.1088/1361-648X/ aa680e,

(36) Aradi, B.; Hourahine, B. dftbplus/dftbplus [DFTB+]: Release 17.1. 2017; https:// doi.org/10.5281/zenodo.809965.

(37) Kubař, T.; Bodrog, Z.; Gaus, M.; Köhler, C.; Aradi, B.; Frauenheim, T.; Elstner, M. Parametrization of the SCC-DFTB method for halogens. J. Chem. Theory Comput 2013, 9, 2939-2949, DOI: $10.1021 /$ ct4001922.

(38) Frisch, M. J., et al. Gaussian 16 Revision B.01. 2016; Gaussian Inc. Wallingford CT.

(39) Grimme, S.; Antony, J.; Ehrlich, S.; Krieg, H. A consistent and accurate ab initio parametrization of density functional dispersion correction (DFT-D) for the 94 elements H-Pu. Chem Phys 2010, 132, DOI: 10.1063/1.3382344.

(40) Papajak, E.; Zheng, J.; Xu, X.; Leverentz, H. R.; Truhlar, D. G. Perspectives on basis sets beautiful: Seasonal plantings of diffuse basis functions. J. Chem. Theory Comput. 2011, 7, 3027-3034, DOI: 10.1021/ct200106a.

(41) Curtiss, L. A.; Redfern, P. C.; Raghavachari, K. Gaussian-4 theory. Chem Phys 2007, 126, 084108, DOI: $10.1063 / 1.2436888$.

(42) Dávalos, J. Z.; Notario, R.; Cuevas, C. A.; Oliva, J. M.; Saiz-Lopez, A. Thermochemistry of halogen-containing organic compounds with influence on atmospheric chemistry. Comput. Theor. Chem. 2017, 1099, 36-44, DOI: 10.1016/j.comptc.2016.11.009. 
(43) Das, S. K.; Chakraborty, S.; Ramakrishnan, R. Critical benchmarking of popular composite thermochemistry models and density functional approximations on a probabilistically pruned benchmark dataset of formation enthalpies. J. Chem. Phys. 2021, 154, DOI: $10.1063 / 5.0032713$.

(44) Arkane: Automated Reaction Kinetics and Network Exploration. https: //reactionmechanismgenerator.github.io/RMG-Py/users/arkane/, Accessed: 2019-01-29.

(45) Burgess, D. R.; Babushok, V. I.; Linteris, G. T.; Manion, J. A. A Chemical Kinetic Mechanism for 2-Bromo-3,3,3-trifluoropropene (2-BTP) Flame Inhibition. Int. J. Chem. Kinet. 2015, 47, 533-563, DOI: 10.1002/kin.20923.

(46) Smith, G. P.; Tao, Y.; Wang, H. Foundational Fuel Chemistry Model Version 1.0. 2016; http://nanoenergy.stanford.edu/ffcm1.

(47) Ruscic, B.; Bross, D. H. Active Thermochemical Tables (ATcT) values based on ver. 1.122p of the Thermochemical Network (2020); available at ATcT.anl.gov. https:// atct.anl.gov/ThermochemicalData/version1.122p/.

(48) Karton, A.; Sylvetsky, N.; Martin, J. M. W4-17: A diverse and high-confidence dataset of atomization energies for benchmarking high-level electronic structure methods. Comp. Chem. 2017, 38, 2063-2075, DOI: 10.1002/jcc.24854. 